J. Clin. Chem. Clin. Biochem.

Vol. 25, 1987, pp. $423-430$

(C) 1987 Walter de Gruyter \& Co. Berlin - New York

\title{
Zur klinischen Wertigkeit der Sialinsäure im Serum bei benignen und malignen Erkrankungen
}

\author{
Von K.-H. Henn und A.M. Gressner \\ Abteilung für Klinische Chemie und Zentrallaboratorium, Klinikum der Philipps-Universität Marburg
}

(Eingegangen am 8. Oktober 1986/4. März 1987)

Zusammenfassung: Die Sialinsäurekonzentrationen im Serum wurden bei benignen und malignen Erkrankungen bestimmt und die Kriterien der diagnostischen Zuverlässigkeit (Spezifität, Sensitivität, Effizienz, likelihood ratio, Vorhersagewert) für beide Gruppen berechnet. Die Sialinsäurekonzentration war bei benignen (Median 0,826 g/l, 5. Perzentil 0,62 g/l, 95. Perzentil 1,27 g/l, p < 0,001) und malìgnen Erkrankungen (Median 0,90 g/l, 5. Perzentil 0,67 g/l, 95. Perzentil 1,48 g/l, p < 0,001) signifikant gegenüber dem Wert der Referenzpopulation (Median 0,62 g/l, 5. Perzentil 0,52 g/l, 95. Perzentil 0,78 g/l) erhöht. Maligne Erkrankungen wiesen gegenüber benignen Erkrankungen signifikant höhere $(p<0,005)$ Sialinsäurekonzentrationen auf. In der Gruppe der benignen Erkrankungen zeigte sich eine besonders starke Erhöhung bei rheumatoider Arthritis und Kollagenosen (Median 1,03 g/l) und bei infektiös-entzündlichen Erkrankungen (Median 0,97 g/l), bei malignen Erkrankungen in der Gruppe der gastrointestinalen Carcinome (Median 1,1 g/l), Blasentumoren ( $=2,1,12 \mathrm{~g} / \mathrm{l}$, $1,08 \mathrm{~g} / \mathrm{l}$ ) und Corpuscarcinome (Median 1,0 g/l). Die Sensitivität beträgt bei einer Entscheidungsgrenze von $0,78 \mathrm{~g} / \mathrm{l}$ für benigne Erkrankungen 0,68, für maligne Zustände 0,83, für beide Krankheitsgruppen gemeinsam 0,71. Die Spezifität beträgt für benigne Erkrankungen 0,94 , für maligne 0,32, für beide Krankheitsgruppen 0,94. Der positive Vorhersagewert bei einer Entscheidungsgrenze von $0,78 \mathrm{~g} / \mathrm{l}$ beträgt für benigne Erkrankungen bei einer angenommen Prävalenz von $0,1(0,2) 0,55(0,72)$, für maligne Erkrankungen bei einer Prävalenz von $0,1(0,2), 0,11(0,23)$, für beide Krankheitsgruppen zusammen bei einer Prävalenz von $0,1(0,2) 0,77$ $(0,88)$. Korrelationen zwischen den Konzentrationen von Sialinsäure und C-reaktivem Protein $\left(r_{s}=0,284\right)$, Fibronectin $\left(r_{s}=0,143\right)$, carcinoembryonalem Antigen $\left(r_{s}=0,367\right)$ und Protein $\left(r_{s}=-0,0166\right)$ bestehen nicht. Verlaufsbeobachtungen von Patienten mit gastrointestinalen Carcinomen weisen deutlich die Überlegenheit des carcinoembryonalen Antigens gegenüber Sialinsäure als Tumorkenngröße aus. Die Sialinsäure nimmt nur relativ geringfügig und sehr langsam nach Resektion des Tumors ab. Aus den erhobenen Daten ist eine diagnostische Validität der Sialinsäure für die allgemeine Diagnostik und Verlaufkontrolle von Tumoren nicht zu erkennen.

\section{The clinical significance of sialic acid in serum in benign and malignant diseases}

Summary: The concentrations of sialic acid in the serum of patients with benign and malignant diseases were determined and the criteria of diagnostic validity (sensitivity, specificity, efficiency, likelihood ratio, predictive values) were calculated. The concentrations of sialic acid in sera of patients with benign diseases (median $0.826 \mathrm{~g} / \mathrm{l}, 5$. percentile $0.62 \mathrm{~g} / 1,95$. percentile $1.27 \mathrm{~g} / \mathrm{l}, \mathrm{p}<0.001$ ) and malignant diseases (median $0.90 \mathrm{~g} / 1,5$. percentile $0.67 \mathrm{~g} / 1,95$. percentile $1.48 \mathrm{~g} / \mathrm{l}, \mathrm{p}<0.001$ ) were significantly higher than those in a population of healthy persons (median $0.62 \mathrm{~g} / 1,5$. percentile $0.52 \mathrm{~g} / \mathrm{l}, 95$. percentile $0.78 \mathrm{~g} / \mathrm{l}$ ). In malignant diseases, the serum sialic acid concentrations are significantly higher $(p<0.005)$ than those in benign diseases. In the group of benign diseases marked increases were seen in patients with rheumatoid arthritis and collagenoses (median $1.03 \mathrm{~g} / \mathrm{l}$ ) and infectious-inflammatory diseases (median $0.97 \mathrm{~g} / \mathrm{l}$ ). In the group of malignant diseases increases were seen in cases of gastrointestinal carcinomas (median $1.1 \mathrm{~g} / \mathrm{l}$ ), bladder 
tumours $(\mathrm{n}=2,1.12 \mathrm{~g} / \mathrm{l}, 1.08 \mathrm{~g} / \mathrm{l})$ and carcinoma of the uterus (median $1.0 \mathrm{~g} / \mathrm{l}$ ). The sensitivity at a decision limit of $0.78 \mathrm{~g} / \mathrm{l}$ for benign diseases is 0.68 , for malignant diseases 0.83 and for both categories 0.71 . The specificity for benign diseases is $\mathbf{0 . 9 4}$, for malignant diseases 0.32 , for both categories 0.94 . For benign diseases, the positive predictive values at a decision limit of $0.78 \mathrm{~g} / 1$ are 0.55 and 0.72 (given prevalences 0.1 and 0.2 , respectively); for malignant diseases, 0.11 and 0.23 (prevalences 0.1 and 0.2 ); for both categories 0.77 and 0.88 (prevalences of 0.1 and 0.2 ). There are no significant correlations between the concentrations of sialic acid and C-reactive protein $\left(r_{s}=0.284\right)$, fibronectin $\left(r_{s}=0.143\right)$, carcinoembryonic antigen $\left(r_{s}=\right.$ $0.367)$, and protein $\left(r_{s}=-0.0166\right)$. Follow up of patients with gastrointestinal carcinoma reveals that carcinoembryonic antigen is much superior to sialic acid as a follow up parameter of tumours. Sialic acid declines only moderately and slowly after resection of the tumour. The data do not suggest that sialic acid is a valuable parameter in the diagnosis and follow up of tumour patients.

\section{Einführung}

Als Sialinsäure werden $\mathrm{O}$ - und $\mathrm{N}$-Acylderivate der Neuraminsäure zusammengefa $B t$, die in $\alpha$-glykosidischer Bindung meist in terminaler Position Bestandteil von Oligo- und Polysacchariden sowie von Glykokonjugaten (Glykoproteinen und -lipiden) sind (1). Während die Konzentration an freier Sialinsäure in Geweben ùnd Körperflüssigkeiten im allgemeinen sehr gering ist, kommt der Zucker als Baustein der Glykokonjugate ubiquitär in intrazellulären Strukturen, Zellmembranen und verschiedenen Körperflüssigkeiten vor (1). Die biologischen Funktionen der Sialinsäure, die teilweise durch ihre physikochemischen Eigenschaften (Hydrophilie, starke Elektronegativität) und durch die terminale Position in Oligosaccharidketten bedingt sind, erscheinen komplex und betreffen unter anderem die Bestimmung der Halbwertszeit von Plasmaglykoproteinen durch Maskierung des präterminalen (rezeptorspezifischen) $\mathrm{Ga}$ lactosylrestes (2), die Beeinflussung der Fluidität von Zellmembranen $(3,4)$ sowie die Maskierung normaler und neoplastischer antigener Strukturen $(5-8)$. Die Konzentration der protein- und lipidgebundenen Sialinsäure wird durch die katalytischen Aktivitäten von Sialyltransferase (EC 2.4.99.1) und Neuraminidase (Sialidase) (EC 3.2.1.18) reguliert. Abgesehen von einigen, meist seltenen hereditären Anomalien des Sialinsäurestoffwechsels $(9-12)$ sind sowohl benigne als auch maligne Erkrankungen bekannt, die zu erhöhten Sialinsäurekonzentrationen in Serum und Urin führen (13-26). Die Bestimmung der Sialinsäure im Serum wurde von einigen Autoren wiederholt als eine für die Diagnostik und Verlaufskontrolle der Progression und Metastasierung von Carcinomen nützliche Laboruntersuchung vorgeschlagen, wobei neben der totalen Sialinsäurekonzentration auch selektiv die lipidgebundene Form gemessen wurde (22, 27-29). Bisher fehlen jedoch quantitative Angaben zur diagnostischen Validität dieser nicht unumstrittenen Kenngröße sowohl für die benignen als auch malignen Erkrankungen. In dieser Studie wurden des:- halb unter Anwendung einer vollmechanisierten, enzymatisch-kinetischen Methode (30) die Konzentrationen der Sialinsäure im Serum von Normalpersonen und Patienten mit diversen benignen und malignen Erkrankungen bestimmt und wichtige diagnostische Kriterien wie Spezifität, Sensitivität, Effizienz, likelihood ratio und Vorhersagewerte für diese beiden Erkrankungsgruppen ermittelt.

\section{Material und Methoden}

Probengewinnung

\section{Referenżkollektiv}

Das dieser Studie zugrunde gelëgte Referenzkollektiv aus klinisch und biochemisch offensichtlich gesunden Probanden entstammat einer früheren Studie (30) und bestand aus 126 Blutspendern im Alter zwischen 19 und 48 Jahren (74 Männer, 52 Frauen). Die Seren wurden bis zur Analyse maximal 1 Monat bei $-20^{\circ} \mathrm{C}$ gelagert.

\section{Patientenkollektiv}

Es wurden 238 frische Patientenseren aus dem Routineprogramm des Żentrallabors des Klinikums hinsichtlich ihrer Sialinsäurekonzentration analysiert. Die klinischen Diagnosen wurden aus den Krankenakten übernommen, anhand derer 192 Patienten den benignen (im Alter von 19 bis 85 Jahren) und 46 den malignen Erkrankungen (im Alter von 16 bis 87 Jahren) zugeordnet werden konnten. Zusätzlich wurden 123 Patientinnen mit gynäkologischen Malignomen mit untersucht. Ưber die Zusammensetzung des Patientengutes mit benignen und malignen Erkrankungen informieren Abbildung 3 und 6.

\section{Analytische Methoden}

Die Sialinsäurebestimmung erfolgte mit einer enzymatischen Methode unter Verwendung von N-Acetylneuraminsäurè-Aldolase (Boehringer Mannheim GmbH). Diese Methode wurde als enzymatisch-kinetisches Verfahren durch Adaptation auf den Cobas Bio Zentrifugalanalysator (Hoffmann LaRoche, Grenz̈ach) vollmechanisiert, die analytischen Kriterien sind berichtet worden (30). Gesamtprotein wurde mit der Biuret-Methode, adaptiert auf den Hitachi 737 (Boehringer Mannheim GmbH), bestimmt. Die Konzentration von C-reaktivem Protein wurde mit einem immunnephelometrischen Latex-Test der Fa. Behring AG, Marburg, die des carcinoembryonalen Antigens mit Hilfe eines Enzymimmunoassays (Firma Abbott, Wiesbaden) ermittelt. 


\section{Statistische Auswertung}

Die Prüfung der Sialinsäurekonzentration im Serum auf Normalverteilung erfolgte graphisch mittels Wahrscheinlichkeitspapier. Korrelationen wurden mit den Spearman-Rangkorrelationskoeffizienten, Signifikanzen mit dem U-Test nach Mann \& Whitney beschrieben (32). Die Berechnungen von Spezifität, Sensitivität, Vorhersagewerten, diagnostischer Effizienz und likelihood ratio basieren auf den allgemein üblichen Definitionen dieser Kriterien (33-35).

\section{Ergebnisse}

Referenzbereich der Sialinsäurekonzentration im Serum

Die Sialinsäurekonzentrationen im Serum gesunder Probanden sind nicht normal verteilt. Der Median des Referenzintervalles wurde mit $0,62 \mathrm{~g} / 1$ bestimmt, das 5. Perzentil liegt bei $0,52 \mathrm{~g} / 1$, das 95 . Perzentil bei $0,78 \mathrm{~g} / \mathrm{l}$. Der Bereich zwischen dem 2,5. und 97,5. Perzentil liegt zwischen $0,51 \mathrm{~g} / 1$ und $0,82 \mathrm{~g} / \mathrm{l}$. Ein signifikanter Geschlechtsunterschied besteht nicht. Die Verteilung der Konzentrationen in der Referenzpopulation zeigt Abbildung 1.

Sialinsäurekonzentrationen im Serum von Patienten mit benignen Erkrankungen

In Abbildung 2 ist dargestellt, daß die Sialinsäurekonzentrationen bei benignen Erkrankungen deutlich erhöht sind. Der Median beträgt 0,826 $\mathrm{g} / 1$ und liegt somit signifikant $(\mathrm{p}<0,001)$ höher als der gesunder Probanden (Abb. 1). Die 5. (2,5.) und 95. (97,5.) Perzentile liegen bei $0,62 \mathrm{~g} / \mathrm{l}(0,57 \mathrm{~g} / \mathrm{l})$ bzw. $1,27 \mathrm{~g} / \mathrm{l}$ $(1,39 \mathrm{~g} / \mathrm{l})$, die gemessenen Konzentrationen erstrecken sich über einen Bereich von $0,49 \mathrm{~g} / 1$ bis $1,56 \mathrm{~g} / \mathrm{l}$. Bei 135/192 (70,3\%) Patienten liegt die gemessene Sialinsäurekonzentration über dem 95. Perzentil, bei 117 (60,9\%) Patienten über dem 97,5. Perzentil. Die höchsten Konzentrationen fanden sich in der Gruppe der rheumatoiden Arthritiden und Kollagenosen (Median 1,03 g/l) sowie bei inffektiös-entzündlichen Erkrankungen (Median 0,97 g/l) (Abb. 3). Bei Patienten mit rheumatoider Arthritis lagen alle Sialinsäurekonżentrationen oberhalb des 95 . Perzentils des Referenzkollektivs. Nur in der Gruppe der Bluterkrankungen (Anämien und Gerinnungsstörungen) lag der Median $(0,67 \mathrm{~g} / \mathrm{l})$ unterhalb des 95 . Perżentils des Referenzkollektivs. Einzelheiten der Verteilung der Sialinsäurekonzentrationen bei verschiedenen Erkrankungen sind Abbildung $3 \mathrm{zu}$ entnehmen. Die Unterschiede in den Medianen der Konzentration der einzelnen benignen Krankheitsgruppen waren im allgemeinen statistisch nicht signifikant.

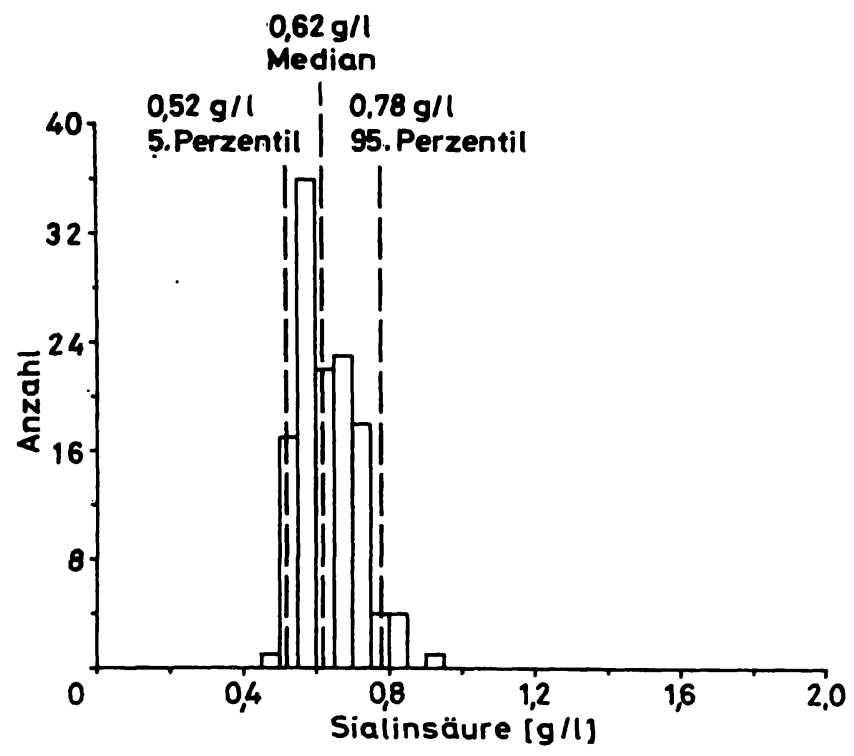

Abb. 1. Histogramm der Sialinsäurekonzentrationen im Referenzkollektiv ( $n=126,74$ Männer, 52 Frauen, Altersbereich 19 bis 48 Jahre).

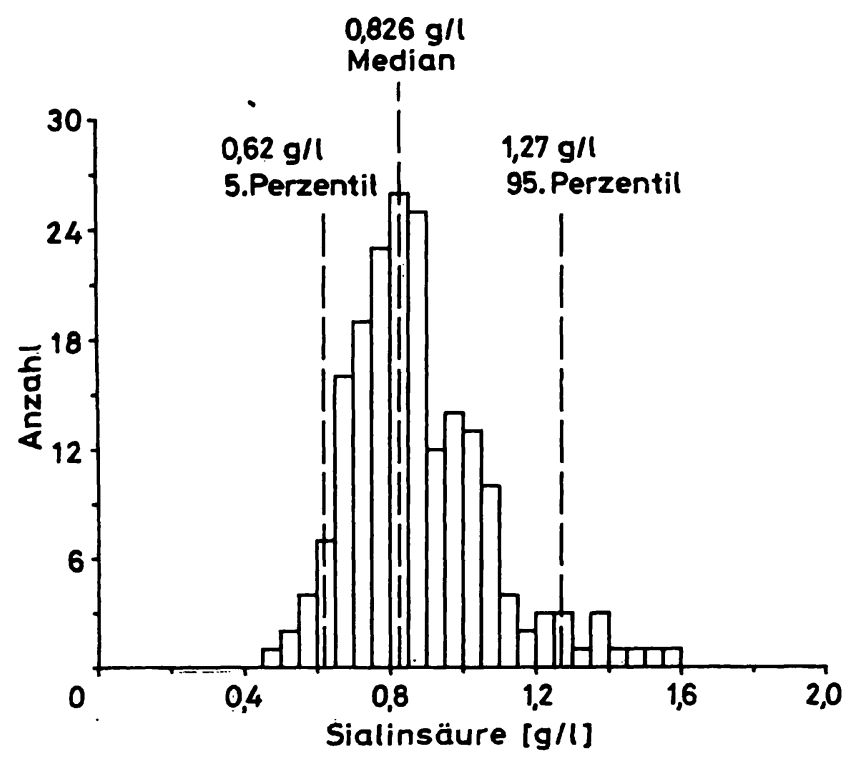

Abb. 2. Histogramm der Sialinsäurekonzentrationen von $\mathrm{Pa}$ tienten mit verschiedenen benignen Erkrankungen (n = 192, 114 Männer, 78 Frauen, Altersbereich 19 bis 85 Jahre).

Die diagnostische Sensitivität erhöhter Sialinsäurekonzentrationen für benigne Erkrankungen beträgt bei einer Entscheidungsgrenze von $0,78 \mathrm{~g} / 1 \mathrm{l}, 68$, die Spezifität 0,94. Weitere Angaben zur Sensitivität, Spezifität, Effizienz und likelihood ratio in Abhängigkeit von der Lage des Entscheidungskriteriums sind in Tabelle 1 enthalten. Bei einer Entscheidungsgrenze von $0,78 \mathrm{~g} / 1$ wurde der positive Vorhersagewert bei einer Prävalenz von $0,1(0,2)$ mit $0,55(0,72)$ bestimmt (Abb. 4). Für benigne und maligne (siehe unten) Erkrankungen liegt der positive Vorhersagewert bei der gleichen Entscheidungsgrenze und Prävalenz von $0,1(0,2)$ bei $0,77(0,88)$. 


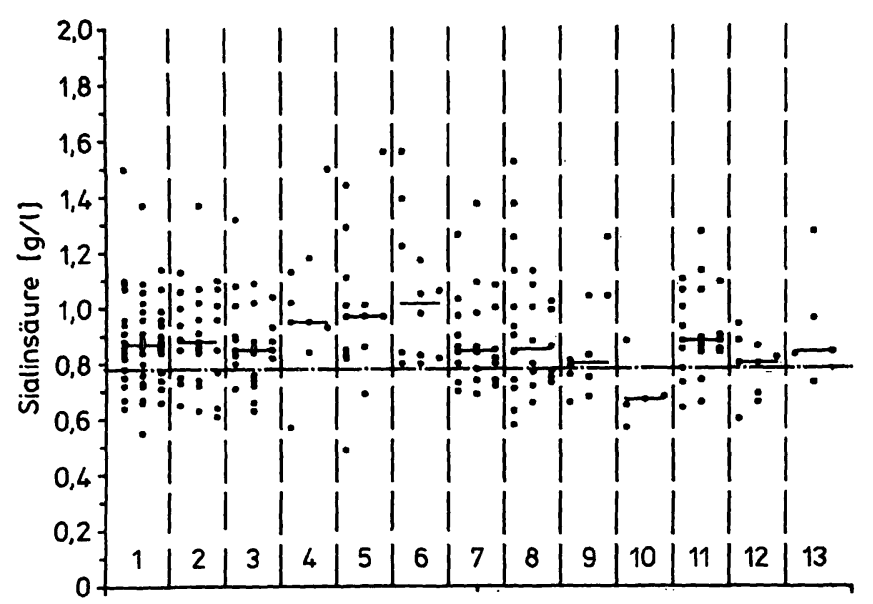

Abb. 3. Verteilung der Sialinsäurekonzentrationen im Serum von Patienten mit verschiedenen benignen Erkrankungen.

Krankheitsgruppen:

1. ischämische Herzerkrankungen, 2. Stoffwechselerkrankungen (Diabetes, Gicht, Hyperlipoproteinämien), 3. Arterielle Verschlußkrankheiten, Hypertonie, 4. Apoplexien, 5. Entzündungen, post operationem, 6.irheumatische Arthritiden, Kollagenosen, Autoimmunerkrankungen 7. Niereninsuffizienz, Nierensteine 8. Gastrointestinalerkrankungen (Ulcera, Cholelithiasis, Leberzirrhose), 9. Skelettsystem (Osteoporose, Arthrose), 10. Blut (Anämien, Gerinnungsstörungen), 11. Lunge (chronisch obstruktive Erkrankungen, Fibrosen), 12. Schilddrüse (Hypo- und Hyperthyreose), 13. Psychosyndrome.

Die Mediane der Konzentrationen sind durch horizontale Balken angegeben, die obere Referenzbereichsgrenze $(95$. Perzentile $=0,78 \mathrm{~g} / \mathrm{l})$ ist durch die gestrichelte Linie gekennzeichnet.

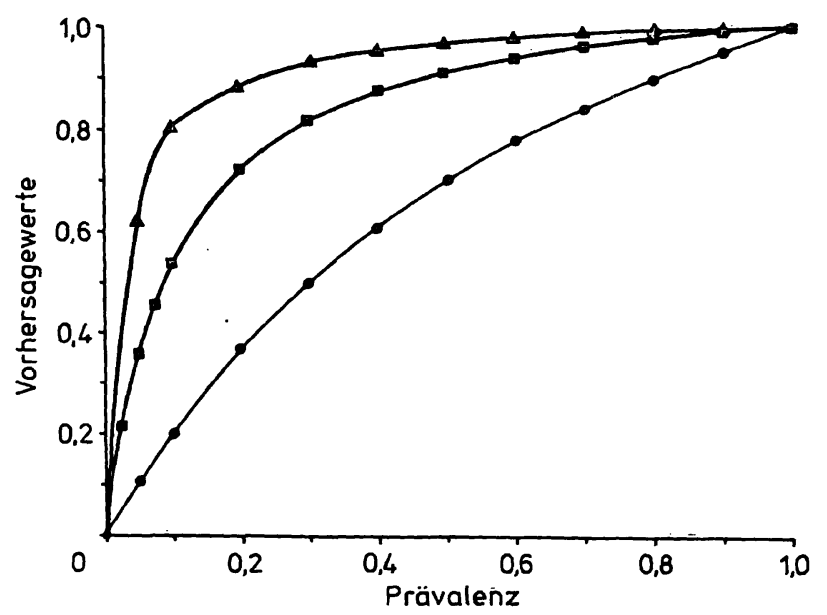

Abb. 4. Vorhersagewerte erhöhter Sialinsäurekonzentrationen im Serum (Entscheidungsgrenze $0,78 \mathrm{~g} / \mathrm{l}$ ) für alle benignen Erkrankungen $(n=192)(\mathbf{m}-\mathbf{a})$ und für die Summe von benignen und malignen Erkrankungen $(n=238)(\Delta-\Delta)$ in bezug auf ein Kollektiv gesunder Probanden $(n=126)$. Vorhersagewerte erhöhter Sialinkonzentrationen $(0,78 \mathrm{~g} / \mathrm{l})$ für alle malignen Erkrankungen $(n=46)(-0)$ beziehen sich auf ein Kollektiv von Patienten mit benignen Erkrankungen.
Sialinsäurekonzentrationen im Serum von Patienten mit malignen Erkrankungen

Die Sialinsäurekonzentrationen bei malignen Erkrankungen sind sowohl gegenüber der Referenzpopulation $(p<0,001)$ als auch gegenüber benignen Erkankungen $(p<0,005)$ signifikant ęrröht. Der Median beträgt $0,90 \mathrm{~g} / \mathrm{l}$, die 5 . $(2,5$.$) und 95. (97,5$.) Perzentile liegen bei $0,67 \mathrm{~g} / 1(0,65 \mathrm{~g} / \mathrm{l})$ bzw. $1,48 \mathrm{~g} / 1(1,79 \mathrm{~g} / \mathrm{l})$ (Abb. 5). Die gemessenen Konzentrationen erstrecken sich über einen Bereich vôn $0,61 \mathrm{~g} / 1$ bis $1,86 \mathrm{~g} / 1$. Bei $84,8 \%$ der Patienten mit malignen Erkrankungen liegt die Sialinsäurekonzentration oberhalb des 95. Perzentils des Referenzkollektivs. Die Mediane der Konzentration von Sialinsäure lagen bei allen Gruppen über dem 95. Perzentil der Referenzgruppe. Die relativ höchsten Konzentrationen fanden sich in der Gruppe der gastrointestinalen Carcinome (Median $1,1 \mathrm{~g} / \mathrm{l})$, bei Blasentumoren $(\mathrm{n}=2,1,12 \mathrm{~g} / 1,1,08$ $\mathrm{g} / \mathrm{l}$ ) sowie bei Corpuscarcinomen (Median 1,0 g/l) (Abb. 6).

Die diagnostische Sensitivität erhöhter Sialinsäurekonzentrationen für maligne Erkrankungen beträgt bei einer Entscheidungsgrenze von $0,78 \mathrm{~g} / 1 \mathrm{l}, 83$, die Spezifität 0,32 (Tab. 1). Angaben zur diagnostischen Effizienz und der likelihood ratio erhöhter Sialinsäurekonzentrationen für maligne Erkrankungen finden sich in Tabelle 1. Bei gleicher Entschcheidungsgrenze wurde der positive Vorhersagewert bei einer Prävalenz von $0,1(0,2)$ mit $0,11(0,23)$ bestimmt (Abb. 4). Bei den Berechnungen wurden Patienten mit benignen Erkrankungen als Bezugskollektiv gewählt.

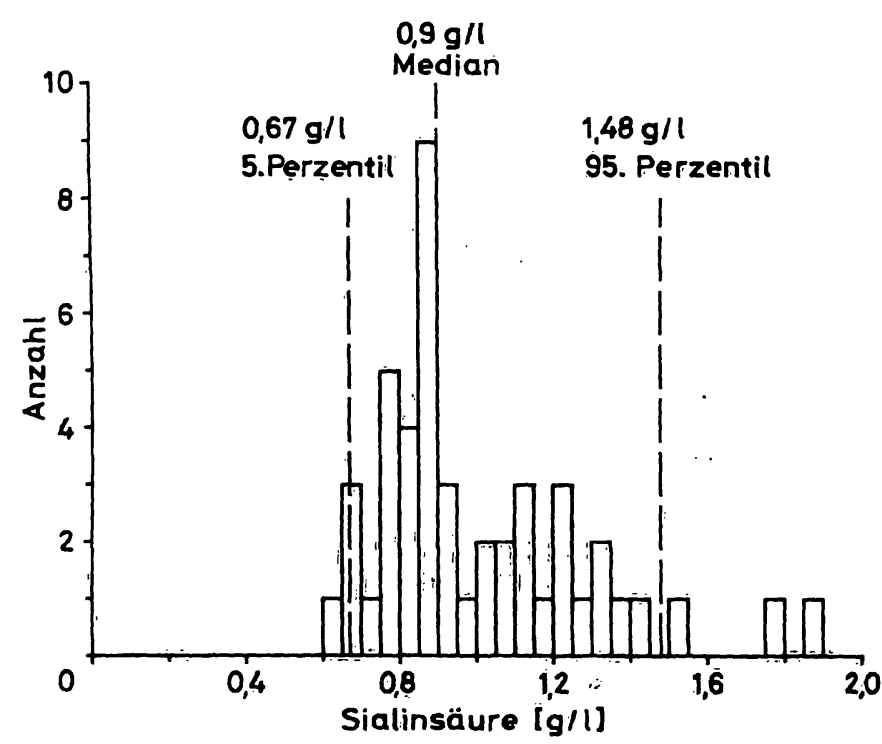

Abb. 5. Histogramm der Sialinsäurekonżentrationen von $\mathbf{P a}-$ tienten mit verschiedenen malignen Erkrankungen $(\mathrm{n}=46$, Altersbereich 16 bis 87 Jahre). 
Tab. 1. Diagnostische Sensitivitäten, Spezifitäten, Effizienzen und likelihood ratios erhöhter Sialinsäurekonzentrationen im Serum bei unterschiedlichen Entscheidungsgrenzen. Die Kriterien für benigne Erkrankungen wurden geprüft mit einem Referenzkollektiv gesunder Probanden, die für maligne Erkrankungen mit einem Bezugskollektiv bestehend aus Patienten mit benignen Erkrankungen.

\begin{tabular}{|c|c|c|c|c|c|c|}
\hline \multirow[t]{2}{*}{ Erkrankungsgruppe } & \multirow{2}{*}{$\begin{array}{l}\text { Diagnostisches } \\
\text { Kriterium }\end{array}$} & \multicolumn{5}{|c|}{ Entscheidungsgrenze (g/l) } \\
\hline & & 0,71 & 0,78 & 0,82 & 0,91 & 0,96 \\
\hline $\begin{array}{l}\text { Benigne } \\
\text { Erkrankungen }\end{array}$ & $\begin{array}{l}\text { Sensitivität } \\
\text { Spezifität } \\
\text { likelihood ratio } \\
\text { Effizienz }\end{array}$ & $\begin{array}{l}0,84 \\
0,79 \\
4,00 \\
0,82\end{array}$ & $\begin{array}{r}0,68 \\
0,94 \\
11,33 \\
0,78\end{array}$ & $\begin{array}{r}0,60 \\
0,98 \\
30,00 \\
0,75\end{array}$ & $\begin{array}{r}0,36 \\
0,99 \\
36,00 \\
0,61\end{array}$ & $\begin{array}{l}0,30 \\
1,00 \\
- \\
0,57\end{array}$ \\
\hline $\begin{array}{l}\text { Maligne } \\
\text { Erkrankungen }\end{array}$ & $\begin{array}{l}\text { Sensitivität } \\
\text { Spezifität } \\
\text { likelihood ratio } \\
\text { Effizienz }\end{array}$ & $\begin{array}{l}0,91 \\
0,16 \\
1,08 \\
0,29\end{array}$ & $\begin{array}{l}0,83 \\
0,32 \\
1,22 \\
0,42\end{array}$ & $\begin{array}{l}0,72 \\
0,40 \\
1,20 \\
0,45\end{array}$ & $\begin{array}{l}0,50 \\
0,64 \\
1,38 \\
0,61\end{array}$ & $\begin{array}{l}0,43 \\
0,70 \\
1,43 \\
0,65\end{array}$ \\
\hline $\begin{array}{l}\text { Benigne und } \\
\text { Maligne } \\
\text { Erkrankungen }\end{array}$ & $\begin{array}{l}\text { Sensitivität } \\
\text { Spezifität } \\
\text { likelihood ratio } \\
\text { Affizienz }\end{array}$ & $\begin{array}{l}0,86 \\
0,79 \\
4,10 \\
0,83\end{array}$ & $\begin{array}{r}0,71 \\
0,94 \\
11,80 \\
0,79\end{array}$ & $\begin{array}{r}0,63 \\
0,98 \\
31,50 \\
0,75\end{array}$ & $\begin{array}{r}0,39 \\
0,99 \\
39,00 \\
0,60\end{array}$ & $\begin{array}{l}0,32 \\
1,00 \\
- \\
0,56\end{array}$ \\
\hline
\end{tabular}

Sialinsäurekonzentrationen im Verlauf maligner Erkrankungen

Bei einer Reihe intestinaler (Colon-, Rectumcarcinom) und extraintestinaler (Mammacarcinom, Meningeom) Neoplasien wurde die Sialinsäurekonzentration im Serum vor und nach chirurgischer Thera-

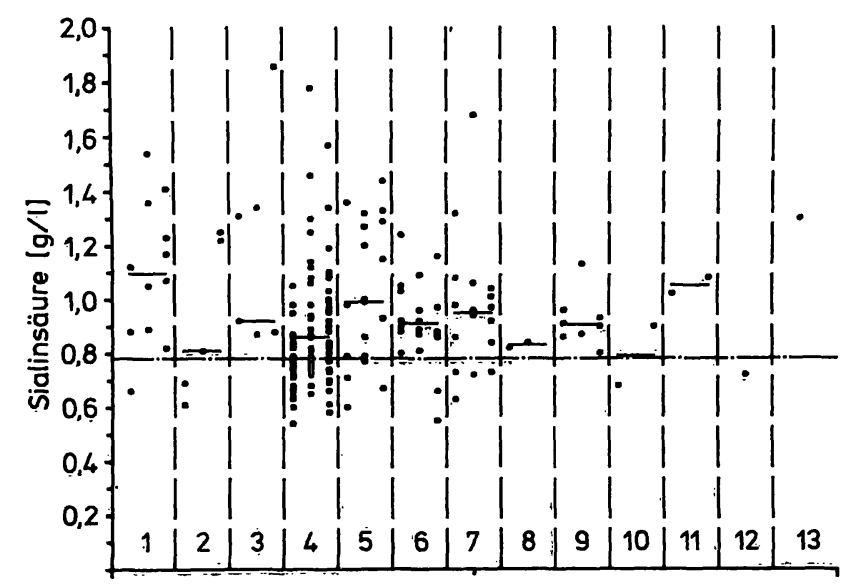

Abb. 6. Verteiluñg der Sialinsäurekonzentrationen im Serum von Patiènten mit verschiedenen malignen Erkrankungen $(n=46)$. Zusätzlich wurden 123 Patientinnen mit gynäkologischen Tumoren in verschiedenen Stadien mit aufgenommen.

Kitankheitsgruppen:

1. Magen-, Colon=, Rectum-Carcinome, 2. Hirntumoren (Gliome, Mèningeome), 3. Bronchialcarcinome, 4. Mammacarcinome, 5. Corpuscarcinome, 6. Collumcarcinome, 7. Ovarialcarcinome, 8. Schilddrüsencarcinome, 9. maligne Lymphome, 10. Prostatacarcinome, 11. Blasencarcinome, 12. Nierencarcinom, 13. Knochensarkom.

Die Mediane der Konzentrationen sind durch horizontale Balken angegeben, die obere Referenzbereichsgrenze $(95$. Perzentil $=0,78 \mathrm{~g} / \mathrm{l})$ ist durch die gestrichelte Linie gekennzeichnet. pie bestimmt. In Abbildung 7 ist der Verlauf der Sialinsäure- und carcinoembryonalen Antigen-Konzentration vor und nach Hemicolektomie bei rechtsseitigem Coloncarcinom dargestellt. Die intial deutlich erhöhte Sialinsäurekonzentration von $1,42 \mathrm{~g} / 1$ fallt innerhalb von 12 Tagen post operationem auf $1,08 \mathrm{~g} / \mathrm{l} \mathrm{ab}$, bleibt jedoch über einen gemessenen Zeitraum von 14 Tagen nach vollständiger Tumorresektion deutlich erhöht. Die Konzentration des carcinoembryonalen Antigens hingegen fällt post operationem auf Referenzwerte ab. Bei einem über einen längeren Zeitraum beobachteten Patienten mit Rectumamputation bei Rectumcarcinom ließ sich auch ein mäßiger Abfall der Sialinsäurekonzentration von initial $1,1 \mathrm{~g} / 1$ auf $0,77 \mathrm{~g} / 166$ Tage nach Operation

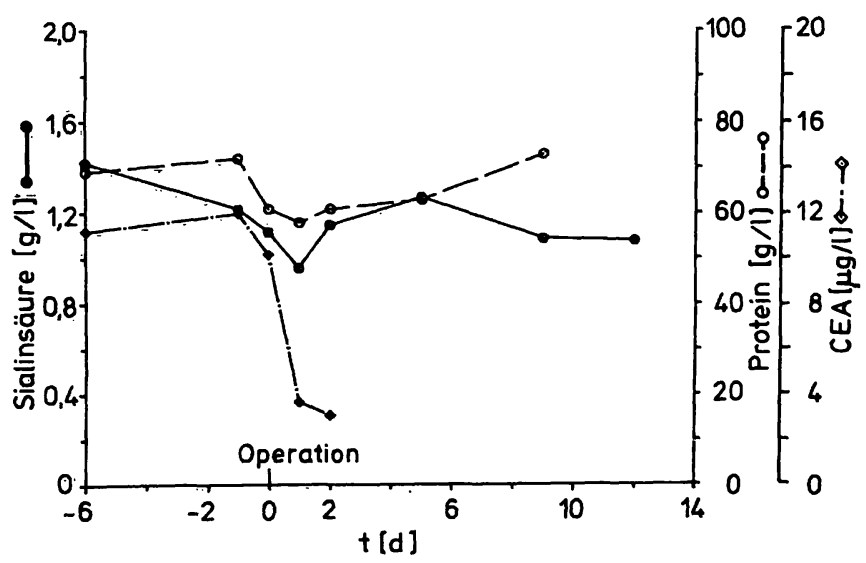

Abb. 7. Verlauf der Konzentrationen im Serum von Sialinsäure $(\bullet-\bullet)$, carcinoembryonalem Antigen $(\diamond-\diamond)$ und Protein $(0-0)$ bei einer 80jährigen Patientin mit Hemicolektomie rechts bei Coloncarcinom. Der Zeitpunkt der Operation ist als Tag 0 bezeichnet. 
feststellen. Auch in diesem Falle reagierte das carcinoembryonale Antigen wesentlich empfindlicher als die Sialinsäure, die auch am Ende des Beobachtungszeitraums noch im pathologischen Grenzbereich lag (Abb. 8). Bei dem dargestellten Verlauf fällt eine unmittelbar postoperativ stark erhöhte Sialinsäurekonzentration bis $1,38 \mathrm{~g} / 1$ auf, die wahrscheinlich auf das Operationstrauma zurückzuführen ist.

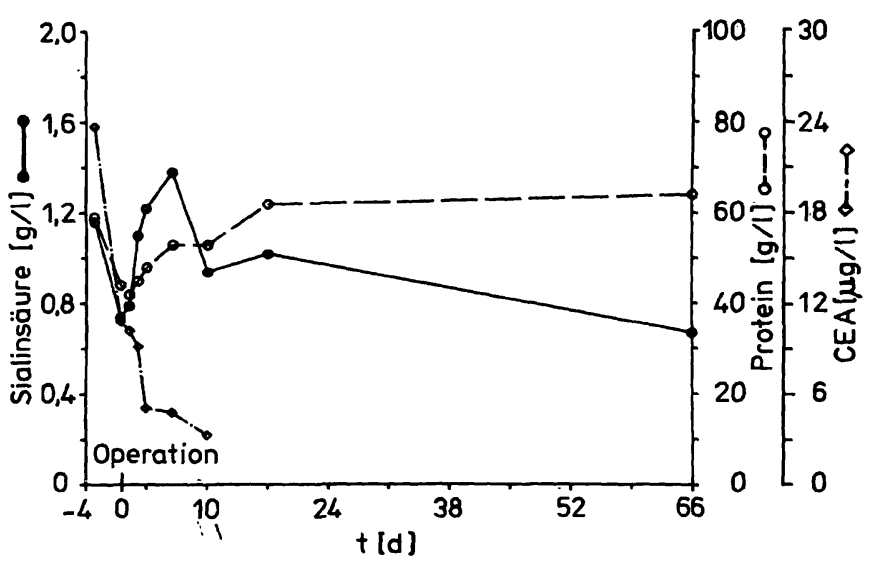

Abb. 8. Verlauf der Konzentration im Serum von Sialinsäure $(\diamond-\diamond)$, carcinoembryonalem Antigen $(\diamond-\diamond)$ und Protein $(0-0)$ bei einer 62jährigen Patientin mit Rectumamputation bei Rectumcarcinom. Der Zeitpunkt der Operation ist als Tag 0 bezeichnet.

Korrelationen $z w i s c h e n$ Sialinsäure und den Konzentrationen von C-reaktivem Protein, carcinoembryonalem Antigen und Fibronectin

Aus den in Tabelle 2 zusammengefaßten Daten ist zu entnehmen, daß die Sialinsäurekonzentration im Serum von Patienten mit benignen und malignen Erkrankungen nicht mit der Konzentration eines typischen Reaktanten der akuten Phase (C-reaktives Protein) oder bei gastrointestinalen Malignomen mit der Konzentration des carcinoembryonalen Antigens korreliert ist. $\mathrm{Zu}$ ähnlichen Aussagen kommen intraindividuelle Verlaufsbeobachtungen (Abb. 7 und 8). Auch zur Fibronectin- und Gesamtproteinkonzentration des Plasmas bestehen keine Korrelationen.

Tab. 2. Korrelationen von C-reaktivem Protein (CRP), Fibronectin, carcinoembryonalem Antigen (CEA) (ausschließlich maligne Erkrankungen) und Protein mit der Sialinsäurekonzentration im Serum. $r_{s}=$ SpearmanRangkorrelationskoeffizient.

\begin{tabular}{lllll}
\hline & $\begin{array}{l}\text { CRP } \\
(\mathrm{mg}(\mathrm{l})\end{array}$ & $\begin{array}{l}\text { Fibronectin } \\
(\mathrm{mg} / \mathrm{l})\end{array}$ & $\begin{array}{l}\text { CEA } \\
(\mu \mathrm{g} / \mathrm{l})\end{array}$ & $\begin{array}{l}\text { Protein } \\
(\mathrm{g} / \mathrm{l})\end{array}$ \\
\hline $\mathrm{n}$ & 23 & 37 & 60 & 125 \\
Bereich & $5,5-120$ & $215,7-861$ & $3,1-590$ & $40-88$ \\
Median & 19,96 & 324 & 13,55 & 71 \\
$\mathbf{r}_{\mathbf{s}}$ & 0,284 & 0,143 & 0,367 & $-0,017$. \\
\hline
\end{tabular}

\section{Diskussion}

Die vorgelegte klinische Studie bestätigt die in früheren Untersuchungen mit anderen Methoden der Sialinsäurebestimmung erhaltenen Ergebnisse, denen zufolge erhöhte Sialinsäurekonżentrationen im Serum bei benignen (vorwiegend entzündlichen) und malignen Erkrankungen nachweisbar sind $(13-29,36)$. Diese Errhöhungen sind weder spezifisch für die maligne oder benigne Erkrankungsgruppe noch lassen sich in der überwiegenden Zahl der Fälle zwischen den einzelnen Erkrankungen in jeder Gruppe signifikante Konzentrationsunterschiede feststellen. Es ist möglich, wurde jedoch der Zielsetzung entsprechend in der vorliegenden Untersuchung nicht geprüft, daß die Höhe der Sialinsäurekonzentration die Aktivität eines entzündlichen Prožesses (z. B. einer rheumatoiden Arthritis) widerspiegelt. Es ließen sich jedoch zwischen den Konzentrationen von Sialinsäure und C-reaktivem Protein, einer klassischen Kenngröße der akuten Phase (37), weder bei benignen noch malignen Erkrankungen signifikante Korrelationen feststellen. Dieses Ergebnis weicht ab von Mitteilungen, die mäBige Korrelationen zwischen Sialinsäure und den Akute-Phase-Reaktanten saures $\alpha_{1}$-Glykoprotein, Haptoglobin und $\alpha_{1}$-Proteinaseinhibitor feștgestellt haben $(14,18)$.

Die mitgeteilten Ergebnisse der transversalen und longitudinalen Untersuchung der Sialinsäurekonzentration im Serum von Tumorpatienten unterstützen nicht die pauschale Auffassung, daß die Bestimmung von Sialinsäure im Serum wertvoll für die Diagnose oder Verlaufskontrolle von Malignomen sei $(13,15$, 21). Wenngleich für eine differenzierte individuelle Bewertung noch detaillierte Untersuchungen in bezug auf Tumormasse, Stadium der Erkrankung und Vorhandensein bzw. Ausdehnung von Metastasen notwendig sind, läßt sich in Übereinstimmung mit früheren Untersuchungen bei Patienten mit anderen Tumorarten (z. B. Melanom) feststellen, daß die Sialinsäurekonzentration in Serum nach operativer Resektion des Tumors nur langsam und geringgradig abfällt (16) und weiterhin keine Korrelation zwischen Sialinsäure und etablierten Tumormarkern wie dem carcinoembryonalen Antigen besteht (19). Bei insgesamt 13 längerfristigen (bis zu 3 Monaten) Verlaufsbeobachtungen gastrointestinaler Tumoren und eines $\mathrm{Me}$ ningeoms vor und nach Resektion ergab sich nur in 2 Fällen (Sigmacarcinom, Meningeom) ein klinisch relevanter Abfall der Sialinsäure um 45\% oder auf Referenzwerte. Inșofern sind unsere Ergebnisse dișkrepant zu den beiden von Shaimberger (21) mitgeteilten Verläufen. Auch in der Diagnōstik von Tumoren erweist sich Sialinsäure im Serum, obwohl im Vergleich zu Patienten mit benignen Erkrankungen signi- 
fikant erhöht, aufgrund sehr geringer prädiktiver Werte und Spezifitäten als ungeeignet, wenn die wichtige Abgrenzung der malignen von den benignen Erkrankungen verlangt ist. So erfüllt Sialinsäure entscheidende, von einem effektiven Tumormarker zu fordernde Kriterien wie Spezifität für Malignome und Normalisierung bzw. Verschwinden nach effektiver Therapie nicht (38). Eine Vielzahl von konkurrierenden benignen pathologischen Zuständen kann die Sialinsäurekonzentration im Serum von Patienten mit Neoplasien unspezifisch und positiv beeinflussen. Die Ursache der Konzentrationserhöhung von Sialinsäure bei Malignom-Patienten ist im einzelnen noch ungeklärt, doch ist diese möglicherweise durch die bei Tumorpatienten wiederholt nachgewiesene Aktivitätserhöhung der Sialyltransferase (EC 2.4.99.1) im Serum $(13,16,39-42)$ oder durch ein "shedding“ von Sialinsäure enthaltenden Glykoproteinen und Glykolipiden $(16,43)$ der an dieser Kohlenhydratkomponente besonders reichen Tumorzelloberfläche (44-46) in die Zirkulation bedingt. Die fehlende Korrelation der Konzentrationen von Sialinsäure und Gesamtprotein im Serum, welches sich aus einem hohen Anteil Sialinsäure-enthaltender Glykoproteine zusammensetzt (2), läßt erwarten, daß ein beträchtlicher Anteil der Neuraminsäure an Glykolipide gebunden ist (27-29). Die selektive Bestimmung dieser bei Tumoren erhöhten Sialinsäurefraktion soll sich für die Diagnostik von Malignomen besser eignen als die Gesamtkonzentration (22).

\section{Schlußfolgerungen}

Zusammenfassend ergibt sich somit eine breite, der ubiquitären Gewebeverteilung entsprechende, unspezifische Erhöhung der Sialinsäure im Serum bei vielen malignen und benignen Erkrankungen, die weder eine diagnostische noch differentialdiagnostische Bedeutung erkennen läßt. Die Ergebnisse können nicht Empfehlungen unterstützen, die Sialinsäure generell als eine nützliche klinische Kenngröße für Tumorscreening oder Tumorüberwachung einzusetzen. Dies schließt nicht aus, daß eine Neuraminsäurebestimmung für spezifische, hier nicht untersuchte klinische Fragestellungen $u$. U. wertvoll sein könnte.

\section{Literatur}

1. Schauer, R. (1982) Adv. Carbohydrate Chem. Biochem. 40, $131-234$.

2. Ashwell, G. \& Morell, A. G. (1974) Adv. Enzymol. 4l, 99-128.

3. Weiss, L. J. (1965) Cell Biol. 26, 735-739.

4. Isaka, S., Hotta, K. \& Kurokawa, M. (1970) Exp. Cell Res. $59,37-42$.

5. Kelley, L. K., King, B. F., Johnson, L. W. \& Smith, C. H. (1979) Exp. Cell Res. 123, 167-176.

6. Chiu, J. \& Drummond, K. N. (1972) Am. J. Pathol. 68, $391-406$.

7. Sedlacek, H. H., Weise, M., Lemmer, A. \& Seiler, F. R. (1979) Cancer Immunol. Immunother. 6, 47-58.

8. Simmons, R. L., Aranha, G. V., Gunnarsson, A., Grage, T. B. \& McKhann, C. F. (1978) In: Immunotherapy of Cancer: Present Status of Trials in Man (Terry, W. D. \& Windhorst, D., eds.) Raven, New York, pp. $123-133$.

9. Lowden, J. A. \& O’Brien, J. S. (1979) Am. J. Hum. Genet. $31,1-18$.

10. Den Tandt, W. R., \& Leroy, J. G. (1980) Hum. Genet. 53, $383-388$.

11. Okada, S., Kato, T. \& Miura, S. (1978).Clin. Chim. Acta $86,159-167$.

12. Okamura-Oho, Y., Yamanaka, T., Suzuki, Y., Akagì, M. \& Kobayashi, T. (1984) Clin. Chim. Acta 144, 263-267.

13. Silver, H. K. B., Karim, K. A., Archibald, E. L. \& Salinas, F. A. (1979) Cancer Res. 39, 5036-5042.

14. Silver, H. K. B., Karim, K. A. \& Salinas, F. A. (1980) Br. J. Cancer $41,745-750$.

15. Silver, H. K. B., Rangel, D. M. \& Morton, D. L. (1978) Cancer 41, 1497-1499.

16. Herrmann, W. P. \& Gielen, W. (1979) Arch. Dermatol. Res. $265,321-329$.

17. Mrochek, J. E., Dinsmore, S. R., Tormey, D. C. \& Waalkes, T. P. (1976) Clin. Chem. 22, 1516-1521.

18. Turner, G. A., Skillen, A. W., Buamah, P., Guthrie, D., Welsh, J., Harrison, J. \& Kowalski, A. (1985) J. Clin. Pathol. 38, 588-592.

19. Hogan-Ryan, A., Fennelly, J. J., Jones, M., Cantwell, B. \& Duffy, M. J. (1980) Br. J. Cancer 41, 587-592.

20. Hogan-Ryan, A. \& Fennelly, J. J. (1981) Eur. J. Cancer Clin. Oncol. 17, 843-844.

21. Shamberger, R. J. (1984) J. Clin. Chem. Clin. Biochem. 22, 647-651.

22. Horgan, I. E. (1982) Clin. Chim. Acta 118, 327-331.

23. Carter, A. \& Martin, N. H. (1962) J. Clin. Pathol. 15, 69-72.

24. Krolikowski, F. J., Renter, K., Waalkes, R. P., Sieber, S. M. \& Adamson, R. H. (1976) Pharmacology 14, 47-51.

25. Brozmanova, E. \& Skrovina, B. (1972) Neoplasma 19 , $115-124$

26. Kiricuta, I., Bojan, O., Comes, R. \& Cristian, R. (1979) Arch. Geschwulstforsch. 49, 106-112.

27. Dnistrian, A. M. \& Schwartz, M. K. (1981) Clin. Chem 27, 1737-1739.

28. Katopodis, N., Hirshaut, Y., Geller, N. L. \& Stock, C. C. (1982) Cancer Res. 42, 5270-5275.

29. Dunzendorfer, U., Katopodis, N., Dnistrian, A. M., Stock C. C., Schwartz, M. K. \& Whitmore, W. F. (1981) Invest. Urol. 19, 194-196.

30. Gressner, A. M. \& Henn, K. H. (1985) J. Clin. Chem. Clin. Biochem. 23, 781-785.

31. Gressner, A. M. \& Wallraff, P. (1980) J. Clin. Chem. Clin. Biochem. 18, 797-805.

32. Sachs, L. (1984) Angewandte Statistik. Springer-Verlag, Berlin, 6. Auflage.

33. Galen, R. S. \& Gambino, S. R. (1975) Beyond normality: the predictive value and efficiency of medical diagnoses. Wiley, New York.

34. Büttner, J. (1977) J. Clin. Chem. Clin. Biochem. 15, 1-12. 
35. Radack, K. L., Rouan, G. \& Hedges, J. (1986) Arch. Pathol. Lab. Med. 110, 689-693.

36. Lipton, A., Harvey, H. A., Delong, S., Allegra, J., White, D., Allegra, M. \& Davidson, E. A. (1979) Cancer 43, 1766-1769.

37. Pepys, M. B. (1981) Lancet $I, 653-657$.

38. Borek, E. (1985) Trends Biochem. Sci. 10, 182-184.

39. Ganzinger, U., Dorner, F., Unger, F. M., Moser, K. \& Jentzsch, K. (1977) Klin. Wochenschr. 55, 553-555.

40. Kessel, S. \& Allen, J. (1975) Cancer Res. 35, 670-672.

41. Henderson, M. \& Kessel, D. (1977) Cancer Res. 39, $1129-1131$.
42. Kessel, D., Samson, M. K. \& Shah, P. (1976) Cancer Res. $38,2132-2134$.

43. Hoon, D. B. S., Ng, S. K. \& Ramshaw, I. A. (1985) Br. J. Cancer 51, 755-781.

44. Yogeeswaran, G. \& Salk, P. L. (1981) Science 212, $1514-1516$.

45. Khadapkar, S. V., Sheth, N. A. \& Bhide, S. V. (19:75) Cancer Res. 35, 1520-1523.

46. Beek van, W. P., Smets, L. A. \& Ëhï̈èlot, P. (1973) Cancer Rẹ̦. 33, 2913-2922.

Prof. Dr. med. A. M. Gressner Klinikum der Philipps-Universität Abt. für Klinische Chemie und Zentrallaboratorium

Baldingerstraße

D-3550 Màrburg 